\title{
Explorations in 3D web cartography
}

\author{
Raluca Nicola \\ ESRI R\&D Center Zurich, Raluca Nicola-rnicola@esri.com
}

Keywords: web-mapping, 3D, state of the art, cartographic representations

\begin{abstract}
Interactive 3D cartography has evolved substantially in the last years. New tools allow cartographers and mapping enthusiasts to create 3D web maps with a minimum effort. Technological advancements allow users to interact with 3D maps on a variety of devices, including their mobile phones or virtual reality glasses. As a result, many interactive 3D maps have been created, from which many insights on their design principles and creation process can be obtained. In this talk I will provide an overview of what is currently possible in 3D cartography and what are the current challenges and possible solutions when creating such maps. More precisely, the specifics of 3D symbology and rendering styles ranging from realistic to abstract will be discussed. In addition, 3D cartography is evolving with a heavy influence from different fields like games industry, art, data visualization or architecture, just to name a few. Hence, examples from some non-conventional 3D maps will be given that allow detecting features that come from such different fields and enhance the maps.
\end{abstract}

\section{References}

Semmo, A., Trapp, M., Jobst, M., Döllner, J., 2015. Cartography-Oriented Design of 3D Geospatial Information Visualization - Overview and Techniques. In: The Cartographic Journal, August 2015.

Sieber, R., Schnürer R., Eichenberger R. and Hurni, L., 2013. The Power of 3D Real-Time Visualization in Atlases Concepts, Techniques and Implementation. In: International Cartographic Conference, Dresden, 2013.

Borden, D., Torguson J., Hodler, T., 2008. Cartography: Thematic Map Design. McGraw-Hill Education; 6th Edition, August 21, 2008. 